\title{
Android-based Diabetes Management System
}

\author{
Morium Akter \\ Department of Computer Science and \\ Engineering, Jahangirnagar University \\ Savar, Dhaka, Bangladesh \\ Department of Computer Science and \\ Engineering, Mawlana Bhashani Science and \\ Technology University, Tangail, Bangladesh
}

\author{
Mohammad Shorif Uddin \\ Department of Computer Science and \\ Engineering, Jahangirnagar University \\ Savar, Dhaka, Bangladesh
}

\begin{abstract}
Diabetes mellitus is a chronic disease, increasing worldwide caused by absolute or relative deficiency of insulin. It results in an excessive amount of glucose in the blood associating with a range of severe complications including renal, cardiovascular diseases and blindness. If the diabetes is controlled properly, the severity of the disease is decreased. For this reason it requires correct educational awareness and routine health check. Doctors help in effective diagnosis and treatment of diabetes which associates high costs. Therefore, a diabetes management system with a friendly user-interface, such as a hand-held device is necessary. Among the hand-held mobile phone operating systems android is the most popular. This research presents the development of an android application for diagnosis and treatment of diabetes as well as diabetes along with hypertension.
\end{abstract}

\section{General Terms}

Applications of Computer Science in Modeling, Bio-informatics et. al.

\section{Keywords}

Diabetes mellitus, management, obesity, diabetes and android.

\section{INTRODUCTION}

Diabetes is rapidly increasing in developing countries including Middle-East, Sub-Saharan Africa, South Asia and Latin America [1], [2]. A study among 191 World Health Organization (WHO) member states in 2000 confirms that $2.8 \%$ people for all age groups had diabetes, and this is expected to be $4.4 \%$ by the year 2030. That is the total number of people with diabetes is projected to rise from 171 millions in 2000 to 366 millions in 2030. Diabetes is reaching epidemic proportions in Bangladesh and in some sectors of our society more than $10 \%$ of people have diabetes [3]. There are severe life threatening complications [4], [5] of diabetes, such as hypoglycemic coma, blurred vision, loss of memory, severe impairment of renal function, insulin allergy, acute neuropathy, etc. Every year, 3.2 million people die worldwide because of diabetes-related causes. For the management of diabetes it requires dietary control, physical exercise, oral hypoglycemic agents and insulin administration.

Hypertension along with diabetes may lead to eye and kidney complications. Hypertension is most common in diabetes that affect $20 \%$ to $60 \%$ patients [6] that depends on obesity, ethnicity and age. Hypertension also increases the risk of microvascular complications like nephropathy and retinopathy [7]. The microvascular and macrovascular complications of diabetes are worsened in patients with hypertension present as comorbidity [8]-[12].

Management of diabetes with or without hypertension requires both lifestyle modifications and pharmacotherapy. For proper management of diabetes it will be beneficial if an automated system is developed. Nowadays, mobile-based system has gained tremendous attractions, as it is very user-friendly. Android is a popular mobile phone operating system. Many researchers have already developed some android-based applications for diabetes management. However, these are not so effective and user-friendly. Therefore, the aim of this research is to develop an android-based application for management of diabetes along with hypertension overcoming the existing inconveniences.

The rest of the paper is organized as follows. Section 2 describes the literature review, Section 3 discusses about the medical knowledge of diabetes, Section 4 describes the diagnosis and management of diabetes, Section 5 presents the proposed system, Section 6 shows experimental results and section 7 draws the conclusion of the paper.

\section{LITERATURE REVIEW}

Rudi [13] designed and implemented an expert-telemedicine system capable of storing clinical data in diabetes management, such as blood glucose measurement, insulin injection doses, hypoglycemic events, dietary intake and exercise activity. The stored records allow the doctors to monitor their patients remotely and to advice the patients about insulin dose. But the system is not automated, as patients need doctors.

Kyung et al. [14] developed a system for diabetic patients consisting of four main menus to manage personal information, diet, exercise, and blood glucose level. Their system can be used as a mobile device within the tele-healthcare system integrated with medical center through Internet.

Ambrosiadou et al. [15] described an expert system for diabetes management by blood glucose monitoring in Greece where diet, life style and administration of insulin differ from Northern European countries. The system addressed only insulin administration not oral drugs.

Akter et al. [16] developed a low-cost automated knowledgebased system that helps in self-diagnosis and management of this chronic disease. The system has an easy computer interface, which performs the diagnostic tasks using rules acquired from medical doctors on the basis of patient data.

Uddin and Akter [17] developed a knowledge-based diagnosis and management system for diabetes mellitus. The implementation of the system is done through a web-based technique, which is workable both in offline and online with a convenient interface.

Blood Glucose Records designed by n225.zero [18] records daily blood glucose levels. Diabetes Management designed by Freshware [19] describes the management of diabetes but does not support for $\mathrm{mmol} / \mathrm{l}$ and have to login in the system.

An android application designed by Rossen Varbanov [20] helps to find blood glucose levels, calculate insulin dosage and 
analyzes the data from manually entry from glucometer readings.

Diabetes Facts designed by NewTips [21] describe about type-1 and type-2 diabetes and add a couple search links on mobile in the form of an icon and bookmark link. Ocmeys [22] designed an android application that describes about the evaluation of diabetes and the symptoms of diabetes. Diabetes eDiary designed by VCA Antech Inc [23] describes a system that track the pet's blood glucose levels, insulin injections and activity of the pet.

Easy Diabetes designed by speed [24] allows the user to control the glucose levels in blood. Diabetes designed by Klimaszewski Szymon [25] describes an android application that keep tracks and analyzes the diabetes data (for example, glucose level) to view interactive graphs or send them directly to the doctor.

MB Diabetes Risk Calculator 1.10 designed by MysticBoard.com [26] helps the user to understand about diabetes, the symptoms of diabetes so that user can take timely action. iTenuto soft [27] designed an android application for Type-1 diabetes calculating a pre-meal insulin dose based on patient's blood glucose and the amount of carbohydrates in patient's meal or snack based on your carbohydrates/insulin ratio.

GExperts Inc [28] designed an application that helps to manage diabetes by tracking various data, such as blood glucose, food, medication, blood pressure (BP), pulse, exercise, weight and a detailed log book with tables and graphs so that patient can share with doctor.

Gordon Wong [29] designed an android application to record blood glucose levels, carbohydrates, insulin dosages and exercise. It works as insulin calculator and email spreadsheet reports to doctors and family.

SIM MOBILE [30] developed an android application to monitor diabetes by tracking glucose, insulin, HbAlc, carbohydrate intake, blood, heart rate, weight, medication and exercise. It shows all data on graphs and adds alarms so that the patients never miss a dose.

TonicMinds [31] developed a system for diabetic patient based on lifestyle, diet and on a clinically validated questionnaire. It gives the information about diabetes risk, body mass index (BMI) of the patient.

SquareMed Software GmbH [32] developed a system to enter blood glucose, dosage of insulin, carbohydrates, blood pressure, pulse, weight and saves time by sending the data directly to doctor. The patient can print or export it directly into their dropbox.

All the systems described above have some limitations. To overcome these limitations we have developed an android application for management of diabetes.

\section{MEDICAL KNOWLEDGE OF DIABETES}

Diabetes mellitus is a chronic disease and common metabolic disorder affecting people of all age groups. It is characterized by hyperglycemia, due to deficiency of insulin. Diabetes is a medical condition that results high level of glucose (sugar) in the blood. This is caused by a deficiency of insulin, which is a hormone secreted by the pancreas. Sugar, starches, and other food are converted into energy needed for daily life by Insulin. The clinical signs seen in diabetes are largely related to the elevated absorptions of blood and urine glucose and the inability of the body to use glucose as an energy source due to the deficiency of insulin [1]-[5], [13].
Diabetes is classified as Type-I (Insulin-Dependent Diabetes Mellitus-IDDM), Type-II (Non-Insulin-Dependent Diabetes Mellitus-NIDDM) and Gestational diabetes mellitus (GDM) [1][5], [13].

Type-I diabetes usually occur in the young, although it can occur at any age, and typically in people who are lean. The pancreas does not able to produce insulin because the insulin-producing cells (b-cells) have been destroyed by the body's immune system. Patient with type-I diabetes are dependent on insulin to survive, so they are called insulin dependent diabetes mellitus (IDDM).

Type-II diabetes mellitus occurs when the body makes enough insulin but cannot use it effectively and more often occur in older people who are obese and had sedentary lifestyles. In many cases symptoms is lower and the disease may remain undiagnosed for many years. Type-II diabetes is often associated with a strong genetic predisposition. Once diagnosed, an improvement may result from weight reduction, dietary modification and increased exercise. Oral hypoglycemic agents and in advanced cases insulin may required.

Gestational diabetes mellitus (GDM) is a condition in which women exhibit high blood glucose levels after conception. A significant portion of this type of diabetes becomes normal after delivery. Once the GDM woman becomes normal, she has increased risk of developing GDM in subsequent pregnancies as well as in later life.

\section{DIAGNOSIS AND MANAGEMENT OF DIABETES}

Diagnosis [14]-[16] is a procedure by which a doctor searches for the reasons of disease that best explains the symptoms of a patient. Our android-based system is designed for performing diagnosis based on patient data where patient data can be demographic or clinical. Demographic data is related to the information such as patient's age, sex, location, income, etc. Clinical data is may be physical signs and laboratory results. Physical signs are those detected by a physical examination of patient, like BMI (body-mass index), pulse rate and blood pressure. Laboratory results are those detected via laboratory tests, like blood test, urine test, etc. The diagnosis of the system is based on the patient data [13]-[16]. For example:

I. Test urine for glucose and ketones.

II. Diabetes exhibits if random or fasting blood glucose level:

$$
\begin{aligned}
& \text { - } \quad \text { Fasting plasma glucose }>=7.0 \mathrm{mmol} / \mathrm{l} \\
& \text { - } \quad \text { Random plasma glucose }>=11.0 \mathrm{mmol} / \mathrm{l} \text {. }
\end{aligned}
$$

Diabetes mellitus can be managed through proper diagnosis and preventive measure. Diet can play a role in the treatment of Diabetes. The following approximate data shows the ways of diabetes management:

- Diet alone $-50 \%$ can be controlled adequately.

- Diet + oral hypoglycemic agent- 20\% - 30\% can be controlled.

- Diet and insulin - 30\% can be controlled

\section{PROPOSED SYSTEM}

The flow diagram of our android-based system for the management of diabetes is shown in Fig 1. 


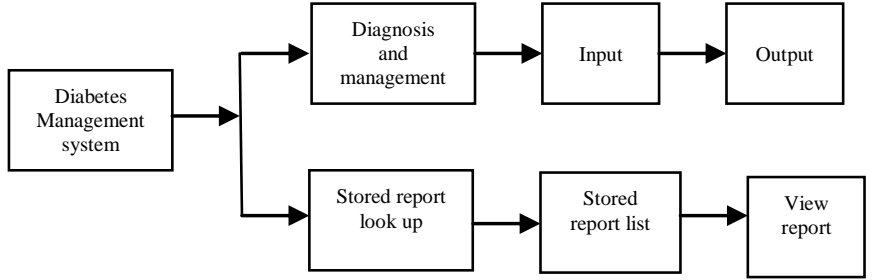

Fig 1: Flow diagram of the proposed system.

The system has two options: one is diagnosis and management of diabetes, another is stored report lookup. In the diagnosis and management option, the system takes user input data for the diagnosis and management. In the stored report lookup option, the system helps in displaying a patient's report.

\section{EXPERIMENTAL RESULTS AND ANALYSIS}

We have implemented our android application for the management of diabetes with hypertension using Eclipse programming language by rule-based approach. Fig 2 presents starting window of the system. Fig 3 to Fig 6 show the experimental results and Fig 7 shows the report.

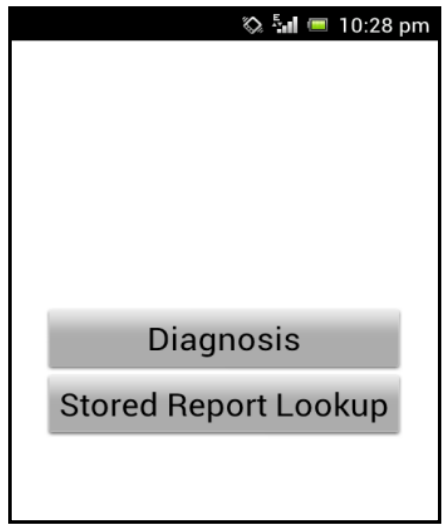

Fig 2 : Starting window of the svstem.

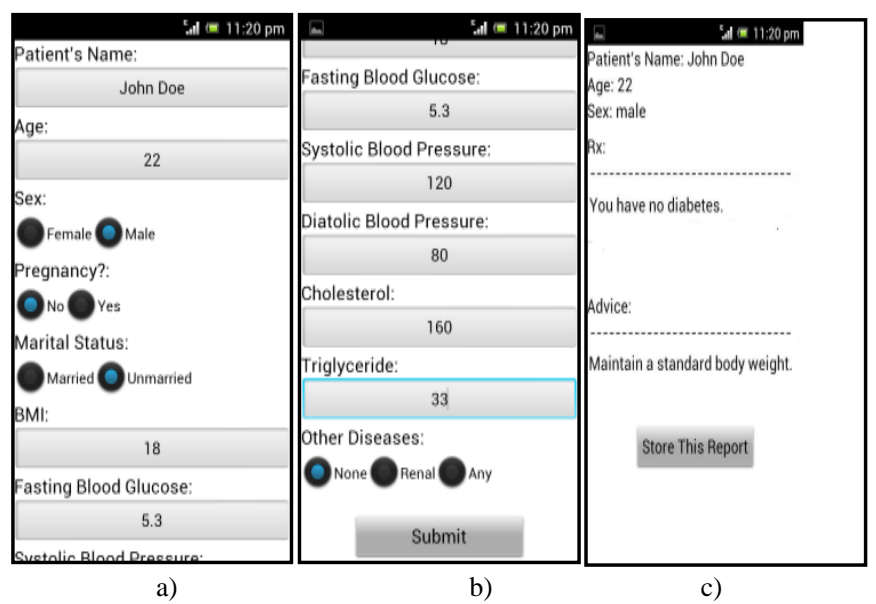

Fig 3: a) input1, b) input2 and c) ouptput(no diabetes)

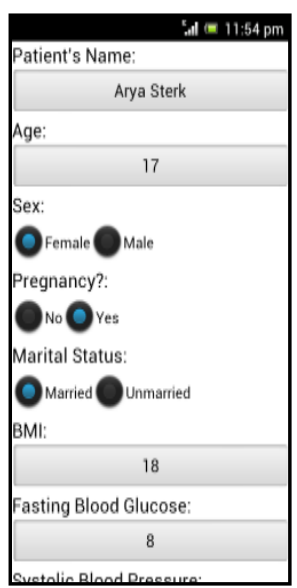

a)

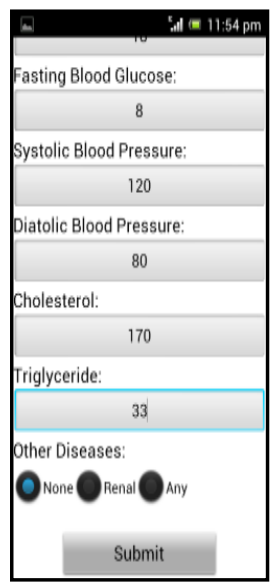

b)

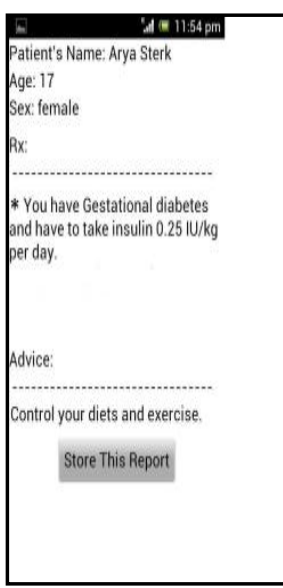

c)
Fig 4: a) input1, b) input2 and c) ouptput(gestational diabetes)
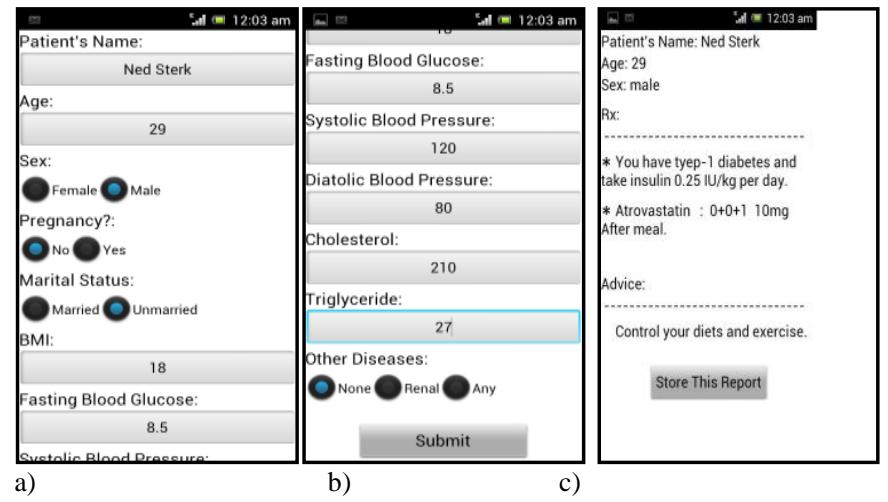

Fig 5: a) input1, b) input2 and c) ouptput(type-1 diabetes)

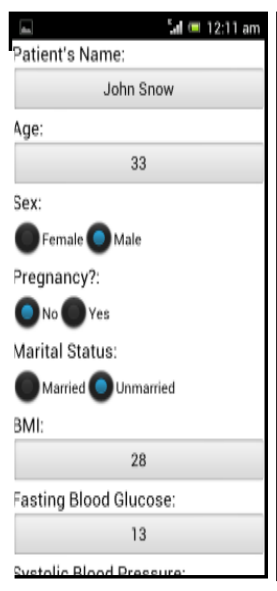

a)

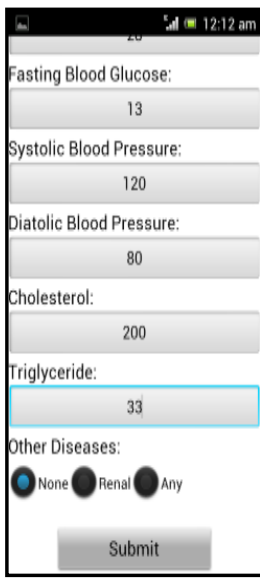

b)

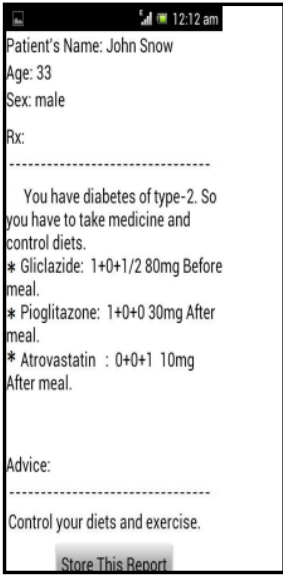

c)
Fig 6: a) input1, b) input2 and c) ouptput(type-2 diabetes) 


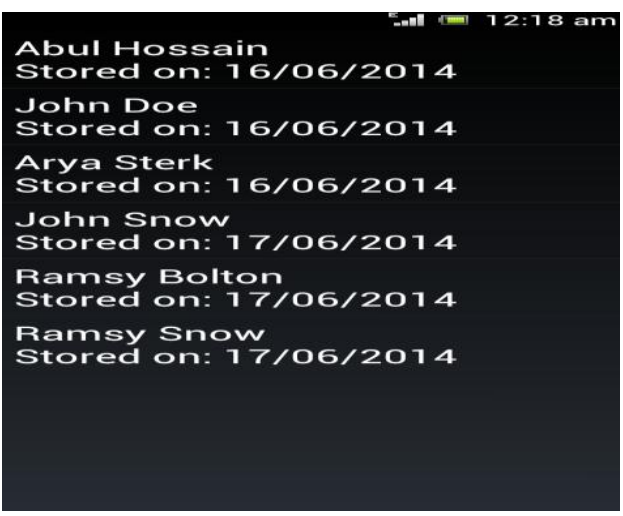

Fig 7: Report view of the system

The system have been tested system using 10 brands of mobile devices (Sony Ericsson, Samsung, Symphony and Micromax) by 50 users and found satisfactory results. A questionnaire was provided to the users about the systems comfortability, usefulness, effectiveness compared to the systems describe in the references [20]-[30]. The performance of the system is shown in Fig 8.

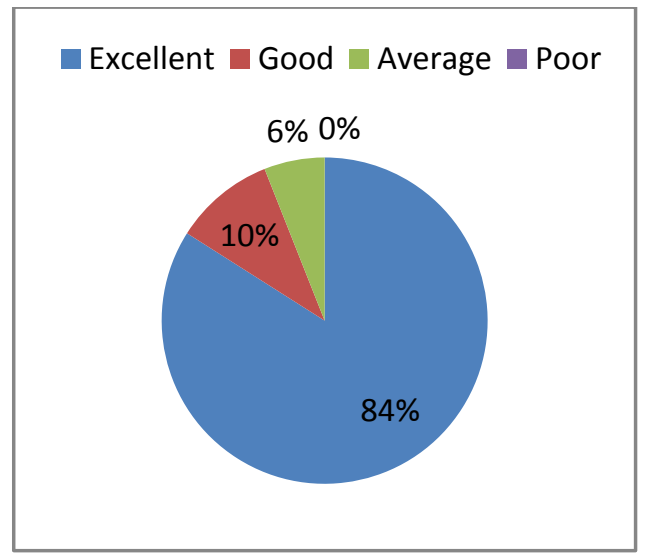

Fig 8: Performance of the system

\section{CONCLUSIONS}

This paper presents the development of an automated android application for the management of diabetes with hypertension. The system is user friendly and has convenient user interface. The reliability, robustness and correctness of the system exact need more elaborate study.

\section{ACKNOWLEDGMENT}

We would like to thank DR. Aminul Haque, Bangladesh Institute of Health Sciences (BIHS) Hospital, Mohammodia Housing Society, Dhaka, Bangladesh, for his sincere cooperation.

\section{REFERENCES}

[1] Afroza Akhter, Kaniz Fatema, Afsana Afroz, Bishwajit Bhowmi, Liaquat Ali and Akhtar Hussain, "Prevalence of Diabetes Mellitus and its Associated Risk Indicators in a Rural Bangladeshi Population," The Open Diabetes Journal, 2011, Volume 4, pp. 6-13.

[2] S. Wild, G. Roglic, A. Green et al., "Global prevalence of diabetes - estimates for the year 2000 and projections for 2030”, Diabetes Care, vol. 27, pp. 1047-1053, (2004).

[3] Diabetic Association of Bangladesh, "Diabetes Mellitus", 2005 (ISBN: 984-32-2552-X).
[4] Sarah Wild, Gojka Roglic, Anders Green, Richard Sicree, Hilary King, "Global Prevalence of Diabetes-Estimates for the year 2000 and projections for 2030", DIABETES CARE, Vol. 27, Number 5, May 2004, pp-1047-1053.

[5] Hajera Mahtab, Zafar A Latif, Md. Faruque Pathan, "Diabetes Melli-tus - A Handbook For Professionals", BIRDEM, 3rd Ed., 2004 (ISBN: 984-31-0100-6).

[6] Square Pharmaceuticals Ltd., Bangladesh vol. 3, no. 1, January-March 2005, "Inside Bangladesh", available online at http://www.squarepharma.com.bd/DiabetesNew sletter/3.1_JanuaryMarch_2005.pdf (accessed on January 22, 2012).

[7] Karenl L. Whalen and Robert D. Steart, "Pharmacologic Management of Hypertension in Patients with Diabetes", available online at http://www.fafp.org/pdf/25.pdf (accessed on August 10, 2013).

[8] MM Rahim, MM Rahman, F Ahmed, J Chowdhury and F Islam, "The prevalence rate of Hypertension in Rural Population of Bangladesh", Journal of Dhaka National Medical College \& Hospital, available online at http://www.banglajol.info/index.php/JDNMCH/article/view $/ 12225$.

[9] Mashitisho MLI, "Management of hypertension in patients with type 2 diabetes mellitus", available online at http://www.safpj.co.za/index.php/safpj/article/download/35 45/4465 (accessed on July 27, 2013).

[10] Anne Belton RN, "Diabetes and Hypertension", available online at http://www.diabetes.ca/documents/forprofessionals/DC--Summer_2010--A.Belton_.pdf (accessed on July 27, 2013)

[11] Lt Col VK Agrawal, Col R Bhalwar, DR Basannar, "Prevalence and Determinants of Hypertension in a Rural Community", available online at http://xa.yimg.com/kq/groups/20489237/1733900229/name /maat08i1p21.pdf(accessed on July 28, 2013)

[12] Stephen W. Durst and Deb Schering, , "Hypertension Management in the Diabetes Patient", available online at http://www.hawaii.edu/hivandaids/Hypertension_Managem ent_in_the_Diabetes_Patient.pdf (accessed on July 29, 2013).

[13] Rudi Rudi, "Design and Implementation of ExpertTelemedicine System for Diabetes Management at Home," available online

at http://www.who.int/diabetes/facts/world_figures/en/(access ed on 28.8.13).

[14] Kyung-Soon Park, Nam-Jin Kim*, Ju-Hyun Hong, MiSook Park, Eun-Jong Cha, Tae-soo Lee, "PDA based Point-of-care Personal Diabetes Management System," Proceedings of the 2005 IEEE Engineering in Medicine and Biology 27th Annual Conference Shanghai, China, September 1-4, 2005, pp. 3749-3752.

[15] B.V. Ambrosiadou, M.Alevizos and G.Ziakas, "Decision Support in Diabetes Management For Optimal Glycaenic Control by Insulin Administration," Systems, Man and Cybernetics, 1993. 'Systems Engineering in the Service of Humans', Conference Proceedings., International Conference on, pp. no. 391-396.

[16] Morium Akter, Mohammad Shorif Uddin, Aminul Haque, "A Knowledge-Based System for Diagnosis and Management of Diabetes Mellitus", 13th International 
Conference on Biomedical Engineering (ICBME2008), Singapore, pp 1000-1003.

[17] Mohammad Shorif Uddin and Morium Akter, "Development of a knowledge-based diagnosis and management system for diabetes mellitus through webbased technique", accepted in ULAB Journal of Science and Engineering, vol.1, 2010.

[18] n225.zero, "Blood Glucose Records" available online at http://www.appbrain.com/app/blood-glucoserecords/com.n225zero.BloodGlucoseRecords (accessed on September 10, 2013).

[19] Freshware, "dbees.com Diabetes Management" available online at http://www.appbrain.com/app/dbees-comdiabetes-management/com.freshware.dbees (accessed on September 10, 2013).

[20] Rossen Varbanov, "My Diabetes", available online at http://www.appbrain.com/app/my-diabetes/com.mydiabetes (accessed on September 10, 2013).

[21] NewTips, "Diabetes Facts," available online at http://www.appbrain.com/app/diabetes-

facts/com.diabetes.test.diabetestest.tips (accessed on September 10, 2013).

[22] Ocmeys, "Diabetes Evaluation," available online at http://www.appbrain.com/app/diabetesevaluation/com.ocmeys.diabetes (accessed on September $10,2013)$

[23] VCA Antech Inc, "Diabetes eDiary," available online at http://www.appbrain.com/app/diabetesediary/air.com.vca.glucosetoolandroid (accessed on September 10, 2013).

[24] speed, "Easy Diabetes," available online at http://www.appbrain.com/app/easy-
diabetes/com.speeD.easydiabetes.main (accessed on September 10, 2013)

[25] Klimaszewski Szymon, "Diabetes," available online at http://www.appbrain.com/app/diabetes/com.szyk.diabetes (accessed on September 10, 2013).

[26] MysticBoard.com "MB Diabetes Risk Calculator 1.10," available online at http://www.freenew.net/windows/mbdiabetes-risk-calculator-110/20879.htm (accessed on September 10, 2013).

[27] iTenuto soft, "Diabetes Personal Calculator," available online at http://www.freenew.net/iphone/diabetes-personalcalculator-12/189758.htm (accessed on September 10, 2013).

[28] GExperts Inc, "OnTrack Diabetes," available online at http://www.appbrain.com/app/ontrackdiabetes/com.gexperts.ontrack (accessed on September 10, 2013).

[29] Gordon Wong, "BG Monitor Diabetes," available online athttp://www.appbrain.com/app/bg-monitordiabetes/com.wonggordon.bgmonitor (accessed on September 10, 2013)

[30] SIM MOBILE , "Diabetes Self Control" available online at http://www.appbrain.com/app/diabetescontrol/com.manishkprr.viewpager (accessed on September 10, 2013).

[31] TonicMinds, "Diabetes Risk Calculator," available online at $\quad \mathrm{http}: / / \mathrm{www}$.freenew.net/android/diabetes-risk calculator/95249.htm (accessed on September 10, 2013).

[32] SquareMed Software GmbH, "DiabetesPlus," available online

http://www.appbrain.com/app/diabetesplus/com.squaremed. diabetesplus.typ1 (accessed on September 10, 2013). 